\title{
A profile of Caesarean sections performed at a district hospital in Tshwane, South Africa
}

\author{
I Govender ${ }^{a *}$, C Steyn $^{a} \mathbb{D}$, O Maphashab ${ }^{b}$ and AT Abdulrazak ${ }^{a}$ \\ ${ }^{a}$ Department of Family Medicine and Primary Health Care, Sefako Makgatho Health Sciences University, Pretoria, South Africa \\ ${ }^{b}$ Department of Family Medicine, University of Pretoria, Pretoria, South Africa \\ *Corresponding author, email: indiran.govender@gmail.com

Introduction: Caesarean section (CS) is a common obstetric procedure that prevents neonatal and maternal death when performed correctly if indicated; however, CS can give rise to complications that lead to maternal and perinatal morbidity and mortality. Rates of CS are increasing worldwide, although the World Health Organization (WHO) has indicated an ideal rate of $5-15 \%$. South African CS rates are higher than the ideal.

Methods: Maternity records of 2015 were reviewed at Odi District Hospital (ODH) to assess whether ODH complies with the ideal CS rate. In this study, extracted data include date and time of CS, maternal age, parity, gestational age, total number of previous CSs, elective or emergency, indications, anaesthesia used and registration of the surgeon.

Results: There were 3336 deliveries and 1064 CSs (32\%). The majority of women were aged from 19 to 34 years (59\%), $72.8 \%$ were multiparous and $54 \%$ between $37-39$ weeks' gestation. The most common (40.1\%) overall and emergency indication was foetal distress. Most CSs were emergencies (61.70\%). Most elective CSs were because of a previous CS and spinal anaesthesia was used in $91.73 \%$. Medical officers performed most of the CSs (79.0\%) during working hours. The CS rate of $32 \%$ was significantly higher than the ideal $5-15 \%$ and higher than in other sub-Saharan countries with similar maternal characteristics. Indications for emergency and elective CSs were similar to previous research.

Conclusion: The Caesarean section rate at $\mathrm{ODH}$ is higher than the recommended rate. Potential CSs therefore need to be evaluated more intensely to assess the true need for surgical intervention.

Keywords: Caesarian section, emergency Caesarian sections, foetal distress, previous caesarian section, cephalo pelvic disproportion

\section{Introduction}

A CS is a common obstetric procedure ${ }^{1}$ performed because of complications during pregnancy, e.g. pre-eclampsia, eclampsia, antepartum haemorrhage, intrapartum foetal distress, cephalopelvic disproportion (CPD), malpresentation, prolonged labour and elective CS for women who had previously undergone the procedure. It is essential to prevent foetal, neonatal and/or maternal death. ${ }^{2} \mathrm{CS}$ rates vary worldwide, ranging from approximately $10 \%$ in Sweden to about $80 \%$ in private-sector hospitals in Brazil. ${ }^{1,3}$ Although the lowest rates of $6.5-7.3 \%$ are seen in Africa, ${ }^{2,4}$ rates of approximately $30 \%$ are reported in regional and tertiary public hospitals in the Durban Metropolitan area of South Africa. ${ }^{1,3}$ CS rates are increasing globally. From 1994 to 2014 the global average increased from $12.4 \%$ to $18.6 \%$, and this upward trend can also be seen in sub-Saharan Africa. ${ }^{2}$

The question has been raised as to whether there is an ideal CS rate. The $\mathrm{WHO}$ established that $\mathrm{CS}$ is an essential procedure in the management of delivery, and recommended a rate of 5$15 \%$ of all births worldwide, based on the CS rates in countries with the lowest perinatal mortality and morbidity. ${ }^{2}$ CS rates higher than $10 \%$ are not associated with a reduction in neonatal and maternal mortality rates. ${ }^{5}$ Caesarean section rates vary depending on a multitude of factors, such as foetal, maternal and medical complications in pregnancy, and cultural attitudes towards $C$. $^{1,3}$

In an audit at a South African private hospital the CS rate was $60.4 \% .^{1}$ The most common indications for a CS in this audit were a previous CS, emergency CS for CPD and elective CS following two or more previous CSs. ${ }^{1}$ These high CS rates are a reflection of the trend in South Africa. A CS rate of $65 \%$ was reported in the private sector in South Africa in 2004, almost double the percentage in the UK and the USA. ${ }^{1,6}$ It was also reported that the equivalent South African public sector figure was estimated to be between $10 \%$ and $20 \%$, which was only marginally higher than that in several leading first-world public health systems, e.g. Sweden, Finland and Norway. ${ }^{1,6,7} \mathrm{~A}$ major South African medical aid scheme reported a CS rate of $63 \%$ of total deliveries performed for its members. ${ }^{1}$

The national CS rate increased in the public health sector, from $18.1 \%$ in $2000 / 2001$ to $24.4 \%$ in $2008 / 2009$ with an annual growth rate of $3.8 \% .^{8}$ Although pregnant women and medical practitioners regard a CS as effective, quick and harmless, ${ }^{9}$ data suggests that morbidity and mortality for mother and foetus/neonate resulting from a CS are higher when compared with vaginal deliveries. ${ }^{1,9,10}$ The risks associated with a CS affect not only the woman, but also her potential child and future pregnancies, and can include short-term and/or longterm risks. ${ }^{5}$

Intraoperative causes of maternal morbidity are haemorrhage due to extension of tears and lacerations, adherent placenta and atonic uterus, and injury to adjacent viscera, difficult intubations and aspiration of gastric contents. ${ }^{11}$ The most common postoperative causes of maternal morbidity are anaemia, urinary tract infections, blood transfusions, prolonged maternal hospital stay due to prolonged catheterisation, infections requiring antibiotics, wound dehiscence and sepsis. The number of women requiring a hysterectomy after a CS is four times higher than for those who deliver vaginally. ${ }^{10,11}$ In countries 
such as Saudi Arabia where large families are encouraged by social and cultural factors, it is not uncommon for women to have as many as six or seven CSs. This high number can lead to uterine scar rupture with significant maternal and foetal morbidity and even mortality. There is, however, no clear evidence to inform clinicians on the number of CSs a woman can safely undergo. $^{12}$

Emergency CSs have shown a rise in severe neonatal morbidity, and an increase in the number of fresh stillbirths and neonatal deaths. Pre-eclampsia is a significant risk factor for maternal death and morbidity. ${ }^{13}$ In sub-Saharan Africa, the highest rates of neonatal morbidity and mortality have been seen in elective $\mathrm{CSs}^{10}$ and are associated with greater risk of neonatal respiratory morbidity and foetal lacerations. ${ }^{14}$ Elective and emergency CSs, however, have reduced the risk of intrapartum foetal death with breech presentations as compared with vaginal delivery. ${ }^{10}$ It seems that poor surgical techniques by junior doctors might also play a role in the CS-associated morbidity and mortality. ${ }^{11}$

At $\mathrm{ODH}$, Pretoria, the theatre and maternity records indicate that the $\mathrm{CS}$ rate at the hospital is increasing. As a result, a retrospective record review was conducted to assess whether $\mathrm{ODH}$ complies with the suggested $15 \%$ by the WHO. We also investigated the characteristics of women undergoing CS and the indications for CS.

\section{Methods}

\section{Study design}

A retrospective record review of CSs at ODH was done.

\section{Study population and sample}

All CSs performed from January 1, 2015 to December 31, 2015 were retrospectively reviewed from records kept at the $\mathrm{ODH}$ maternity unit.

\section{Study setting}

ODH is located in Mabopane, a town close to the border of North West province and $45 \mathrm{~km}$ north of Tshwane in Gauteng Province. $\mathrm{ODH}$ is a 227-bed hospital that includes a labour ward, postnatal ward and theatre facilities. CSs are performed by family medicine registrars, medical officers (full-time and sessional doctors), and doctors doing their year of community service post-internship. Labour ward and theatre staff record every delivery.

\section{Data collection}

Data were collected from the records kept in the labour ward and theatre. The following data were collected: date and time of the CS; maternal age; parity; gestational age in weeks; number of previous CSs; whether the CS was elective or emergency surgery; indication for the CS; whether general or spinal anaesthesia was used; and surgeon's registration (specialist, registrar, medical officer or community service doctor).

\section{Data analysis}

Data was recorded on an Excel spreadsheet (Microsoft Corp, Redmond, WA, USA) and analysed using descriptive statistics. The SPSS statistical software package (version 25; IBM Corp, Armonk, NY, USA) was used to analyse data. Univariate analysis of socio-demographic variables was done. Bivariate analysis was used to determine whether any associations existed between the variables. Data were verified by two researchers who collected identical data independently. An independent data capturer recoded the data onto an Excel spreadsheet; thereafter a statistician analysed the data.

The inclusion of all records for a 12-month period reduced problems of seasonal variation and theatre inactivity for a few weeks.

\section{Ethical considerations}

The Sefako Makgatho University Research Ethics Committee (SMUREC) provided ethical clearance for the research project, clearance number SMUREC/M/303/2016:IR. Permission to conduct the study was obtained from the Odi District Hospital management. Permission and ethical clearance was obtained from the Tshwane Research and Ethics Committee (TREC), project number 54/2016.

\section{Results}

There were 3332 deliveries and 1064 CSs (32\%) at ODH from January to December 2015 and the monthly breakdown is shown in Figure 1.

\section{Maternal characteristics}

The majority of the women were between 19 and 34 years (59\%) of age and the majority were multiparous as indicated in Table 1.

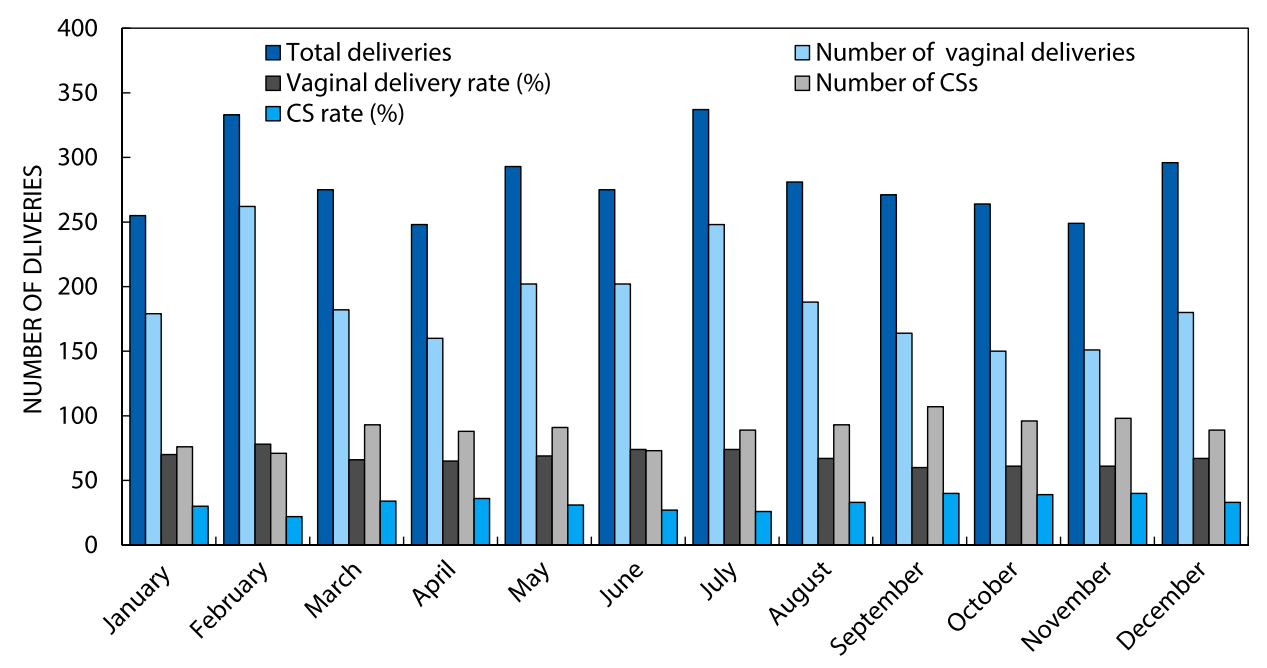

Figure 1: Monthly deliveries in 2015. 
Table 1: Maternal characteristics

\begin{tabular}{lrc}
\hline Maternal characteristic & No. & $\%$ \\
\hline Age (years): & 193 & \\
$<18$ & 617 & 18.1 \\
$19-34$ & 254 & 58.0 \\
$>35$ & & 23.9 \\
Parity: & 289 & \\
Nulliparous & 775 & 27.2 \\
Multiparous & & 72.8 \\
Gestational age (weeks): & 2 & \\
$<27$ & 89 & 0.2 \\
$27-33$ & 170 & 8.4 \\
$34-36$ & 578 & 16.0 \\
$37-39$ & 225 & 54.3 \\
$>39$ & & 21.2 \\
Co-morbidities: & 71 & 6.7 \\
HIV/AIDS & 67 & 6.3 \\
HIV/AIDS on ARV & 64 & 6.0 \\
Hypertension & 6 & 0.7 \\
Diabetes mellitus & & 0.6 \\
Anaemia & 7 & \\
\hline
\end{tabular}

\section{Indications for CS}

The most common indication for CS was foetal distress (40.1\%), which also accounts for most of the emergency CSs performed during the period of our study. One previous CS plus other factors such as having a fresh CS scar, an estimated big baby or poor progress of labour were the reasons for most of the elective CSs as indicated in Table 2.

\section{Surgical procedures}

The majority of CSs were performed as emergency procedures $(61.70 \%)$ and spinal anaesthesia $(91.73 \%)$ was the mode of anaesthesia for most of these. Medical officers performed most of the CSs $(840,79.0 \%)$ and most were done during working hours as evident in Table 3.

Table 2: Indications for CS in 2015

\begin{tabular}{|c|c|c|c|c|}
\hline \multirow[b]{2}{*}{ Indications } & \multicolumn{2}{|c|}{$\begin{array}{l}\text { Emergency } \\
\text { CS }\end{array}$} & \multicolumn{2}{|c|}{ Elective CS } \\
\hline & No. & $\%$ & No. & $\%$ \\
\hline Foetal distress/compromise & 427 & 40.1 & 0 & 0 \\
\hline Cephalo-pelvic disproportion (CPD) & 101 & 9.5 & 0 & 0 \\
\hline $\begin{array}{l}\text { One previous CS + other factors (e.g. } \\
\text { fresh scar, big baby, poor progress) }\end{array}$ & 48 & 4.5 & 106 & 9.9 \\
\hline Failed induction of labour & 23 & 2.2 & 0 & 0 \\
\hline Multiple gestations & 18 & 1.7 & 42 & 3.9 \\
\hline Malpresentation & 17 & 1.6 & 59 & 5.5 \\
\hline Maternal chronic conditions: & 12 & 1.1 & 35 & 3.3 \\
\hline \multicolumn{5}{|l|}{ Hypertension } \\
\hline \multicolumn{5}{|l|}{ HIV/AIDS } \\
\hline \multicolumn{5}{|l|}{ Diabetes mellitus } \\
\hline \multicolumn{5}{|l|}{ Anaemia } \\
\hline Macrosomia & 8 & 0.8 & 28 & 2.6 \\
\hline Two or more previous CS & 0 & 0 & 95 & 8.9 \\
\hline Post dates & 0 & 0 & 45 & 4.2 \\
\hline Total CS (1 064) & 654 & 61.5 & 410 & 38.5 \\
\hline
\end{tabular}

Table 3: Surgical procedure

\begin{tabular}{|c|c|c|}
\hline Variable & No. & $\%$ \\
\hline \multicolumn{3}{|l|}{ Type: } \\
\hline Emergency & 654 & 61.5 \\
\hline Elective & 410 & 38.5 \\
\hline \multicolumn{3}{|l|}{ Mode of anaesthesia: } \\
\hline Spinal anaesthesia & 976 & 91.7 \\
\hline General anaesthesia & 88 & 8.3 \\
\hline \multicolumn{3}{|l|}{ Surgeon's level of experience: } \\
\hline Community service doctor (4) & 65 & 6.1 \\
\hline Medical officers (35 including sessional doctors) & 840 & 79.0 \\
\hline Family medicine registrars (15) & 159 & 15.0 \\
\hline \multicolumn{3}{|l|}{ Time of surgery: } \\
\hline During working hrs (8:00 am-4:00 pm) & 789 & 74.1 \\
\hline After hrs (4:00 pm-8:00 am), weekends, public holidays & 275 & 25.9 \\
\hline
\end{tabular}

\section{Maternal and neonatal complications}

The most significant recorded complication was the admission of $3.4 \%$ of neonates for morbidity reasons. Details of the morbidity were not recorded. Maternal complications were minimal, with post-partum haemorrhage as the most significant recorded maternal complication, as seen in Table 4.

\section{Discussion}

Our study shows CS rates of 32\% during 2015. This is higher than those seen in other sub-Saharan countries, and is significantly higher than the suggested $5-15 \%$ of the WHO. The WHO stated that 'every effort should be made to provide Caesarean sections to women in need, rather than striving to achieve a specific rate. ${ }^{5}$ However, current CS rates worldwide also remain higher than those considered to be medically justifiable. ${ }^{7}$

According to data from 150 countries worldwide, $18.6 \%$ of all births occur by $\mathrm{CS}$, ranging from $6 \%$ in the least developed areas to $27.2 \%$ in the most developed regions. ${ }^{7}$ In Latin America and the Caribbean CS rates are at $40.5 \%$, the highest in the world, with Africa at $7.3 \%$ the lowest. Even though the rates in Africa are low, an increase of $4.5 \%$ has been seen since 1990, while worldwide over the same period the increase was $12.4 \%{ }^{7}$

A study done in Pakistan showed a CS rate of $45.5 \%,{ }^{15}$ and in south-western China the rate was $40.3 \%{ }^{16}$ In China the medical system is strongly influenced by patient preference, in contrast to practices in developed countries. In addition, at the time of our research (2015) China had a one-child policy, which placed healthcare providers there under threat should obstetric outcomes be unsatisfactory ${ }^{16}$

Table 4: Maternal and neonatal complications post CS

\begin{tabular}{lcc}
\hline Maternal complications & No. & $\%$ \\
\hline Septic wound & 1 & 0.1 \\
Wound dehiscence & 2 & 0.2 \\
Post-partum haemorrhage & 4 & 0.4 \\
\hline Neonatal complications & No. & $\%$ \\
\hline Stillbirth & 1 & 0.1 \\
Neonate transferred to tertiary care (indication not & 1 & 0.1 \\
recorded) & & \\
Neonate admitted for morbidity & 36 & 3.4
\end{tabular}


In sub-Saharan Africa CS figures remained mainly unchanged from 1990 to $2014 .^{7}$ It was also seen that target CS rates were met in sub-Saharan Africa between 2010 and 2011. ${ }^{17}$ Although lower rates of CS are desirable, this lack of increase could also indicate a lack of resources and health system deficiencies in these countries; it could also indicate a poor quality of care in this region. ${ }^{7}$

The vast majority of women in our study were between 19 and 34 years of age, in general reflecting the international trend with the exception of Nigeria. In Nigeria more women aged from 35 to 45 years had CSs, as compared with women aged 25-34 and $17-24,{ }^{18}$ whereas in Cameroon, the mean age of the mothers was $26.4 \pm 5.5$ years. ${ }^{19}$ In Mozambique the age group that underwent most CSs was between 20 and 25 years. ${ }^{20}$

In our study $54 \%$ of women were between 37 and 39 weeks' gestation, and $21 \%$ were more than 39 weeks. In private practice in South Africa, the mean gestational age for elective and emergency CS was 38 weeks. ${ }^{1}$ In Cameroon $90 \%$ of CSs were performed at term, ${ }^{19}$ and in Saudi Arabia the gestational age at CS delivery was between 37 and 39 weeks. ${ }^{12}$ In sub-Saharan Africa one third of elective CSs were done at a gestational age of less than 39 weeks. ${ }^{2}$ The probability of antepartum stillbirth with an elective CS has been calculated at $0.08 \%$ at 38 weeks' gestation, and this figure increases to $0.34 \%$ at 41 weeks' gestation. ${ }^{14}$ Infants delivered by pre-labour CS between 37 and 42 weeks gestational age were nearly seven times more likely to develop respiratory morbidity than infants who were delivered vaginally. The rate of respiratory morbidity, however, decreases by 40 weeks' gestational age with elective CS delivery. To minimise potential neonatal risks with an elective $\mathrm{CS}$, this procedure should not be performed prior to a 39-week gestational age. ${ }^{14}$

Our study shows that $1 \%$ of women who underwent emergency $\mathrm{CS}, 3 \%$ of whom had elective CS, had an underlying medical condition as the reason for the CS (Table 2). In this study, a maternal condition was not one of the five greatest indications for either emergency or elective CS. Diabetes mellitus, HIV/AIDS, hypertension and anaemia were the conditions noted in our research.

With a national estimated prevalence of $12.2 \%$, HIV represents the primary burden of disease in South Africa. The annual incidence among people of child-bearing age between 15 and 49 years is estimated at $1.9 \%$, and $2.3 \%$ among people between 15 and 24 years of age. ${ }^{21}$ However, progress has been made in the prevention of mother-to-child transmission of HIV (PMTCT) with transmission rates as low as $2.7 \%$ in 2011. HIV testing of pregnant women is close to $100 \%$ at almost all facilities in South Africa. ${ }^{22}$ In our study $6.7 \%$ of the women who requested CS were tested HIV-positive as seen in Table 1, a figure that is almost half the national estimated prevalence, and $94 \%$ of those women were on anti-retroviral therapy (ART). These results could indicate the fact that an HIV-positive status is not per se an indication for $\mathrm{CS}$ at $\mathrm{ODH}$. On the other hand, it does indicate that the test-and-treat policy for ART is not followed strictly for all pregnant HIV patients, due to the fact that not all HIV-positive women were on ART. In specialist private practice in South Africa, $80 \%$ of HIV-positive patients have an elective CS, while $20 \%$ have an emergency CS due to their positive status. ${ }^{1}$ The motivation for this decision could be that mother-to-child transmission of HIV is reduced with a CS as compared with a normal delivery, but this is debatable in patients with viral loads that are lower than detectable limits. ${ }^{1}$
Hypertension was present in $6 \%$ of women who underwent CS at $\mathrm{ODH}$ as seen in Table 1, but was the reason for CS in a few of the patients. A hypertensive disorder is not only the cause of significant maternal and perinatal morbidity, but is also one of the most common direct causes of maternal mortality. Hypertensive disorders include chronic hypertension, pre-eclampsia, eclampsia and haemolysis, elevated liver enzymes, and low platelet count (HELLP) syndrome. A district hospital should be able to manage women with gestational hypertension, as well as women with mild to moderate pre-eclampsia. Patients with acute severe hypertension, severe pre-eclampsia, imminent eclampsia or eclampsia should, however, be stabilised at the district hospital, and then be referred to a specialist centre. ${ }^{23}$ In subSaharan Africa pre-eclampsia and eclampsia have been given as indication for CS in $2.4 \%$ of patients. ${ }^{17}$ These findings are supported by studies in Pakistan and India, where eclampsia was the indication for $2.09 \%$ and $5 \%$ respectively. No other hypertensive disorders were mentioned in either of these studies. ${ }^{11,15}$

Only $0.7 \%$ of patients in this study had co-morbid diabetes mellitus, and $0.6 \%$ had anaemia. Pregnant women with diabetes mellitus should be referred to specialist health facilities. Follow-up care may be continued at a district hospital in accordance with instructions from the specialist facility. ${ }^{23}$ This arrangement could account for the low number of pregnant patients with diabetes mellitus who underwent $\mathrm{CS}$ at $\mathrm{ODH}$.

Anaemia in pregnancy is due mostly to an iron deficiency. Only $0.6 \%$ of the women in this study had co-morbid anaemia, as presented in Table 1. Multiple factors can contribute to iron deficiency in pregnancy, such as poor diet and parasitic infestations, e.g. schistosomiasis and hookworm. Iron deficiency can be pre-existing, or can occur for the first time due to increased iron demands in pregnancy. Other causes of anaemia in pregnancy include folate deficiency and anaemia of chronic disease such as HIV/AIDS. Malaria is an important cause of anaemia during pregnancy in malarial areas. ${ }^{23}$ In a study done in India $4 \%$ of the women had anaemia, ${ }^{24}$ and in Nigeria $8 \%$ of CS patients were anaemic. ${ }^{18}$ No further details on the cause of the anaemia are provided in either of the studies, or in the $\mathrm{ODH}$ study.

Almost $73 \%$ of women in our study were parous. In Pakistan, $62.3 \%$ of women who requested CS were parous. ${ }^{15}$ These results are confirmed in African studies where only $7.8 \%$ of primigravidas in Nigeria underwent $\mathrm{CS}^{18}$ while in Mozambique mostly parous women underwent $\mathrm{CS}^{20}$ These figures confirm the high CS rates in women who underwent previous CSs.

Emergency CSs comprised $61.5 \%$ of the total CSs performed in our study, with the balance $(38.5 \%)$ being elective procedures. In this study the five most important indications for emergency CS were foetal distress, CPD, one previous CS with other reasons, multiple gestations and malpresentation. The indications for elective CS were one previous CS, two or more previous CS, malpresentation, post-dates and multiple gestations. In sub-Saharan Africa $75-96 \%$ of CSs are reported as emergency procedures, mostly due to seven maternal complications: obstructed labour; prolonged labour; poor presentation; previous CS; foetal distress; uterine rupture; and antepartum haemorrhage (abruptio placenta and placenta praevia). ${ }^{4,17}$ Foetal distress was the most significant foetal reason for an emergency $\mathrm{CS}^{17}$ A multicentre prospective study done in Latin America showed $41.5 \%$ elective CSs. ${ }^{10}$ In South Kerala, India, $88.7 \%$ of CSs were emergency procedures, with $11.3 \%$ being electives. ${ }^{24}$ 
In Pakistan the biggest indication for CS was a previous CS, and $51.5 \%$ of CSs in this country were emergencies. ${ }^{15}$ In Cameroon $12 \%$, and in the UK $7.3 \%$ of CSs were elective procedures done on maternal request. ${ }^{19,24}$ The balance of the Cameroonian CSs $(18 \%)$ were emergency procedures. ${ }^{19}$ In countries like Nigeria, where the CS rate was as low as $7.2 \%$ in one study, the vast majority of the procedures were emergencies. Factors that play a role here are poor infrastructure and cultural preferences that prevent women from opting for an elective $\mathrm{CS}^{18}$ When a woman undergoes an emergency CS the major threats are complications of anaesthesia and surgery, while procedures are often performed in deficient circumstances. During elective CS the mother is prepared preoperatively, and the theatre and staff are adequately prepared. Maternal and foetal complications are therefore more common during emergency $\mathrm{CS}^{11}$ Factors that lead to maternal death during CS were parity $>6$, uterine rupture, antepartum haemorrhage, and preeclampsia/eclampsia. ${ }^{17}$ On the other hand, cord prolapse, antepartum haemorrhage and uterine rupture were the leading causes of early neonatal death. ${ }^{17}$

Spinal anaesthesia was given in $91.7 \%$ of the CSs in our study, as compared with $8.3 \%$ given general anaesthesia. In a WHO survey on maternal and perinatal health in Africa, epidural anaesthesia was given to $0.1 \%$ of the women, without any mention of spinal or general anaesthesia. ${ }^{13}$ In an audit of CSs done in Pakistan, spinal anaesthesia was given to all women included in the audit ( $n=3555$ ) over a six-month period. ${ }^{15}$ It has been reported that the mortality rate of CSs done under general anaesthesia is $16.7 \%$ higher than with regional anaesthesia. Persistent wound pain is less with regional anaesthesia, and anaesthesia-related problems, e.g. airway management, prolonged periods of preoperative fasting and effects of anaesthetic agents on the foetus are lessened when utilising spinal anaesthesia in obstetric surgery. ${ }^{25}$

Family medicine registrars performed $15 \%$ of the CSs in this study, whereas $79 \%$ were done by medical officers and $6 \%$ by community service doctors. Very little information is available on the amount of training that is required to develop proficiency in performing a skilful CS. In many communities, family physicians are the major providers of perinatal care, including CSs, and the need for family physicians with the ability to perform CS is on the increase. It has been shown that family physicians in rural areas provide high-quality $\mathrm{CS}$, and the outcomes of the CS performed by family physicians are similar to those done by obstetricians, but with lower rates of CS. Yet whether these results could be extrapolated to family physicians in training is not known. ${ }^{26}$ However, many countries in sub-Saharan Africa face a critical shortage of healthcare workers and specialists, especially in rural areas. This necessitates medical officers performing a large number of CSs in this region. The indications for CS performed by medical officers are similar to those performed elsewhere in the world, i.e. CPD, previous CS, foetal distress, etc. ${ }^{27}$ It is possible that a lack of surgical skills on the part of medical officers and junior doctors contributes to maternal and neonatal morbidity and mortality post-CS. ${ }^{11}$

Worldwide normal vaginal deliveries occur around the clock and on all days of the week, but it should be noted that few CSs are performed after hours or over weekends. ${ }^{6,28}$ Our study supports this finding, with $74.1 \%$ of the procedures performed during working hours, and only $25.9 \%$ done after hours. This is despite the fact that $61.5 \%$ of the CSs are reported as emergency procedures. A study done in Brazil indicates that more CSs were done on Tuesdays and Wednesdays, and fewer than expected births occurred over weekends. This is in contrast to vaginal births that did not vary according to the day of week. ${ }^{28}$ The pattern regarding time of day was also similar in this study, where the number of CS deliveries between midnight and 6:00 am was less than a quarter of those done between noon and 6:00 pm. There was also a significant reduction in the number of CSs performed during lunchtime. ${ }^{28}$

\section{Limitations}

This record review was done at ODH. The rate of CSs in this institution might not be a true reflection of the overall rate in the district. This descriptive study does not provide information of why the CS rate is high at this district hospital.

\section{Conclusion}

The CS rate at $\mathrm{ODH}$ is higher than the $5-15 \%$ recommended by the WHO. The majority of women who underwent CS were parous women between 19 and 34 years of age, with a gestational age of 37-39 weeks. The majority (61.5\%) of the CSs performed were emergencies, and the five most important indications for emergency CS were foetal distress, CPD, one previous CS with other reasons, multiple gestations and malpresentation. Spinal anaesthesia was given in more than $90 \%$ of CS cases, and although medical officers performed most of the procedures, family medicine registrars may be better equipped to do so. Most CSs were performed during office hours, which could raise the question as to whether emergency CSs are over-diagnosed.

\section{Recommendations}

The indications for CS found in this study seem to be appropriate according to the literature discussed. A potential CS therefore should be evaluated more intensely to assess the true need for surgical intervention. Evaluation could aid in reducing the number of CSs done at ODH to the $10 \%$ recommended by the WHO. This would be in the interests of maternal and neonatal well-being.

Acknowledgements - Mr Stevens Kgoebane assisted with the statistical analysis.

Disclosure statement - No potential conflict of interest was reported by the authors.

\section{ORCID}

C Steyn (iD) http://orcid.org/0000-0001-6062-8981

AT Abdulrazak (D) http://orcid.org/0000-0002-8735-8982

\section{References}

1. Naidoo RP, Moodley J. Rising rates of Caesarean sections: an audit of Caesarean sections in a specialist private practice. S Afr Fam Pract. 2009;51(3):254-8.

2. Harrison MS, Goldenberg RL. Cesarean section in Sub-Saharan Africa. Matern Health Neonatol Perinatol. 2016;2(6). https://doi.org/10.1186/ s40748-016-0033-x.

3. Nathan R, Rautenbach PGDW. Differences in the average Caesarean section rate across levels of hospital care in Gauteng, South Africa. S Afr J Infect Dis. 2014;29(4):147-50. [Cited 2016 Sep 8]. Available from: www.sajei.co.za.

4. Dumont A, De Bernis L, Bouvier-Colle $M H$, et al. Caesarean section rate for maternal indication in Sub-Saharan Africa: a systematic review. Lancet. 2001;358:1328-33.

5. WHO. Statement on Caesarean section rates. Department of Reproductive Health and Research. WHO 2015. [Cited 2018 Oct 2]. Available from: www.who.int/reproductivehealth.

6. Bateman C. Rendering unto Caesar? SAMJ. 2004;94(10):800-2. 
7. Betran AP, Ye J, Moller A, et al. The increasing trend in Caesarean section rates: global, regional and national estimates: 1990-2014. PLoS One. 2016;11(2):e0148343.

8. Monticelli F. Caesarean section delivered in public sector hospitals in South Africa, 2001-2009. Wits Institutional Repository Environment on DSpace. 2013. [Cited 2016 Sep 8]. Available from: www. wiredspace.wits.ac.za.

9. Victora CG, Barros FC. Beware: unnecessary Caesarean sections may be hazardous. Lancet. 2006;367:1796-7.

10. Villar J, Carroli G, Zavaleta N, et al. Maternal and neonatal individual risks and benefits associated with Caesarean delivery: multicentre prospective study. BMJ. 2007;335:1025-36. https://doi.org/10.1136/ bmj.39363.706956.55.

11. Ghazi A, Karim F, Hussain AM, et al. Maternal morbidity in emergency versus elective Caesarean section at tertiary care hospital. J Ayub Med Abbottabad. 2012;24(1):10-3.

12. Sobande A, Eskandar M. Multiple repeat Caesarean sections: complications and outcomes. J Obstet Gynaecol Can. 2006;28:193-7.

13. Shah A, Fawole B, M'Imunya JM, et al. Caesarean delivery outcomes from the WHO global survey on maternal and perinatal health in Africa. Int J Gynae Obstet. 2009; https://doi.org/10.1016/j.ijgo.2009. 08.013.

14. Signore C, Klebanoff M. Neonatal morbidity and mortality after elective Cesarean delivery. Clin Perinatol. 2008;35(2):361-6.

15. Sajjad R, Ali CA, Zia-UI-Haq M. An audit of Caesarean sections in military hospital Rawalpindi. API Care. 2014;8(2):172-5.

16. Ma R, Lao T, Sun Y, et al. Practice audits to reduce Caesareans in a tertiary referral hospital in South-western China. Bull World Health Organ. 2012;90:488-94.

17. Chu K, Cortier H, Maldonado F, et al. Cesarean section rates and indications in Sub-Saharan Africa: a multi-country study from medecins sans Frontieres. PLoS One. 2012;7(9):e44484. [Cited 2019 Feb 12]. https://doi.org/10.1371/journal.pone.0044484.

18. Gunn JKL, Ehiri JE, Jacobs ET, et al. Prevalence of Caesarean sections in Enugu, Southeast Nigeria: analysis of data from the healthy beginning initiative. PLoS One. 2017;12(3):e0174369. [Cited 2019 Sep 8]. https://doi.org/10.1371/journal.pone.0174369.

19. Njim T, Choukem S, Mbu R. Caesarean sections in a secondary level care hospital of Cameroon: an analysis of their six-year trends and adverse neonatal outcomes. BMC Res Notes. 2017;10:430.

20. Long $Q$, Kempas $T$, Madede $T$, et al. Caesarean section rates in Mozambique. BMC Pregnancy Childbirth. 2015;15:253.

21. Department of Health, Republic of South Africa. National HIV testing services: Policy; 2016.

22. Department of Health, Republic of South Africa. National consolidated guidelines for the prevention of mother-to-child transmission of HIV (PMTCT) and the management of HIV in children, adolescents and adults. 24 December 2014.

23. Department of Health, Republic of South Africa. Guidelines for maternity care in South Africa. A manual for clinics, community health centres and district hospitals. 4th ed; 2015.

24. Moni M, Thanganador AT, Yesubaktan SJ. A study on obstetric profile of mothers undergoing primary Caesarean section and their neonatal outcome in a tertiary care centre, South Kerala. Int J Biomed Adv Res. 2015;6(12):835-8.

25. Sumikura $H$, Niwa $H$, Sato $M$, et al. Rethinking general anesthesia for Caesarian section. J Anesth. 2015. https://doi.org/10.1007/s00540015-2099-4.

26. Deutchman M, Connor P, Gobbo R. Outcomes of Caesarean sections performed by family physicians and the training they received: a 15year retrospective study. J Am Board Fam Pract. 1995;8(2):81-90.

27. Chilopora G, Pereira C, Kamwendo F, et al. Postoperative outcomes of Caesarean sections and other major emergency obstetric surgery by clinical officers and medical officers in Malawi. Hum Resour Health. 2007;5(17):1-6.

28. Barros AJD, Santos IS, Matijasevich A, et al. Patterns of deliveries in a Brazilian Birth Cohort: almost universal Caesarean sections for the better-off. Rev Saude Publica. 2011;45(4):635-43.

Received: 31-07-2019 Accepted: 20-09-2019 